Research Article

\title{
An Adjustable Zero Vibration Input Shaping Control Scheme for Overhead Crane Systems
}

\author{
Abdullah Mohammed $\left(D,{ }^{1}\right.$ Khalid Alghanim, ${ }^{1}$ and Masood Taheri Andani ${ }^{2}$ \\ ${ }^{1}$ Department of Mechanical Engineering, Kuwait University, Kuwait City, Kuwait \\ ${ }^{2}$ Center for Vehicle Systems \& Safety, Blacksburg, VA, USA \\ Correspondence should be addressed to Abdullah Mohammed; abdullah@ku.edu.kw
}

Received 27 November 2019; Revised 15 February 2020; Accepted 16 May 2020; Published 28 May 2020

Academic Editor: Chengzhi Shi

Copyright (c) 2020 Abdullah Mohammed et al. This is an open access article distributed under the Creative Commons Attribution License, which permits unrestricted use, distribution, and reproduction in any medium, provided the original work is properly cited.

\begin{abstract}
This article presents a modified zero vibration (ZV) input shaping technique to address the sensitivity and flexibility limitations of the classic ZV shapers commonly implemented in overhead crane applications. Starting with the classical ZV formulation, new parameters are introduced to optimize the control system performance according to a versatile objective function. The new shaper enhances the design flexibility and operational domain of the shaper, while it inherits the robustness properties and computational efficiency of the ZV scheme. Unlike the original ZV shaper, the proposed shaper allows for the point-to-point maneuver time to be fixed. The sensitivity analysis of the controller confirms that the new shaper effectively reduces the ZV sensitivity to the cable length variations.
\end{abstract}

\section{Introduction}

Overhead cranes are widely used in manufacturing plants, nuclear facilities, and shipping yards, where heavy payloads need to be transferred [1]. Although fast crane operations help improving the overall productivity, increasing the maneuver speed can lead to unsafe payload oscillation and residual sway. Therefore, numerous research studies have explored different robust control solutions for these structures. In addition to robustness and swing reduction capabilities, an overhead control scheme must be able to reject external disturbances and function at different crane configurations and loading conditions [2,3]. Moreover, a crane controller performance should be intuitive for a human user to deploy it [4].

Researchers have extensively studied the application of various closed-loop control solutions for payload oscillation suppression in crane systems [5-7]. Gholabi et al. [8] proposed a sensorless control approach based on real-time crane demand. Fuzzy logic and data-driven predictive models were also implemented for sway reduction [9]. Chen et al. [10] developed an adaptive tracker based on a shaped energy-like Lyapunov function that was confirmed to be asymptotically stable and improved the controller robustness in the presence of parameter uncertainties. In a recent study, Zhang et al. [11] evaluated an error-tracking controller platform to minimize the residual sway in a crane subjected to parameter uncertainties and external disturbances. Yang et al. [12] designed an adaptive controller to effectively control the state variables of a ship-mounted crane under harsh operating conditions. Command smoothing control was also proven to be effective for a crane machine with a distributed-mass payload subjected to external force (e.g., wind) [13]. Although most of the antisway control solutions have been developed for point-to-point maneuvers, researchers have also designed and tested controllers that engage in emergency conditions to safely bring the overhead system to a full stop condition [14]. In addition to conventional feedback strategies, optimizationbased control schemes, particularly model prediction control (MPC), have been recently applied to overhead crane platforms. MPC provides solutions for an optimal set of states that minimize the constructed objective function over a receding prediction horizon. The formulation of this 
function determines the type and severity of the system constraints that need to be satisfied by the controller. Schindele and Aschemann [15] developed a nonlinear MPC to minimize the tracking error at crane rest conditions. Smoczek and Szpytko [16] proposed a soft-constrained MPC to minimize the oscillation of a scaled crane setup. In a recent study, the researchers implemented an MPC-based subcontroller to optimize the velocity of the crane cart throughout the prediction horizon with the desired objective of minimizing the payload oscillation [17].

Introduced by Singer et al. in 1990 [18], input shaping has proven to be a powerful control tool to reduce vibrations in dynamics applications. While the effectiveness of feedback controllers strongly relies on the availability of state measurements, input shapers do not require continuous sensing/feedback. Therefore, they are simpler to use and less expensive to implement for overhead crane platforms in industrial environments $[19,20]$. So far, different classes of shapers have been developed and used for crane applications [21-24]. The zero vibration (ZV) method was among the first generations of these input shaping techniques. Despite their robustness [25], ZV shapers are generally associated with slow response times. Therefore, more intricate input shaping strategies, including zero vibration and minimum integral (ZVI) and minimum vibration and integral (MVI), were later developed to offer adaptivity in maneuver time setting, crane cable length, and input commands [26]. Distributed zero vibration (DZV) shapers were also formed by introducing a distributed delay for real-time crane control use cases [27]. Mohammed et al. [28] studied the application of genetic algorithms to optimize the acceleration profiles for a nonlinear input shaper for a crane with strict motion and safety constraints. By optimizing the acceleration input, the optimized shaper provided a faster system response, while satisfying the system constraints. Ramli et al. [29] trained a neural network to operate a real-time unity magnitude zero vibration (UMZV) shaper based on an artificial neural network. Although the experimental analysis confirmed the applicability of this method to crane applications with variable parameters, its implementation highly depends on the availability of adequate data sets for network training. Recently, output-based command shaping (OS) techniques were investigated for sway oscillation caused by hoisting and external disturbances, such as wind forces [30].

In this paper, inspired by the standard ZV formulation, a new shaper is proposed with a flexible objective function that allows the controller to optimize and fix the maneuver time. The existing ZV-based shapers provide limited control over the maneuver time, input acceleration, input jerk, and cable length sensitivity. However, the new shaper can optimize the maneuver time while satisfying the system jerk and sensitivity requirements. This method allows for the input function to be adjusted according to the predefined objective for different operating cases. Simulations are conducted to evaluate the performance of the implemented methodology in mitigating the payload sway and addressing the parameter uncertainties. Moreover, various operating conditions including different cable lengths are investigated. Finally, the effectiveness of the proposed controller is studied in terms of dimensionless metrics, which represent the sensitivity and control jerk.

\section{Methodology}

A standard overhead crane model consists of a massless jib moving in a unidirectional motion along the $x$-axis with displacement function $u$ and an inelastic attached cable of constant length $l$. The payload is represented as a lumped mass $m$ swings in the $x-y$ plane, as shown in Figure 1 .

The kinetic energy $T$ and potential energy $V$ of the system are given by

$$
\begin{aligned}
& T=\frac{1}{2} m\left(\dot{u}^{2}+l^{2} \dot{\theta}^{2}-2 l \dot{u} \dot{\theta} \cos \theta\right), \\
& V=-m g l \cos \theta,
\end{aligned}
$$

where $g$ is the gravity constant and $\theta$ is the swing angle. Using Lagrange's equations to derive the equation of motion of the system gives

$$
\begin{aligned}
\ddot{\imath}+g \sin \theta & =\ddot{u}, \\
\theta(0) & =0, \\
\dot{\theta}(0) & =0 .
\end{aligned}
$$

Assuming a small oscillating angle of the mass $m$ reduces equation (2a) to the linearized equation of motion of the system as

$$
\ddot{\theta}+\omega_{n}^{2} \theta=\frac{\ddot{u}}{l},
$$

where $\omega_{n}$ is the natural frequency of the system, $\omega_{n}=\sqrt{g / l}$. Considering that the jib acceleration $(\ddot{u})$ is a piecewise function of four steps, each of $\Delta t$ duration, the general solution of equation (3) by applying the Duhamel Integral becomes

$$
\begin{aligned}
\theta(t) & =\frac{1}{\omega_{n} l} \int_{0}^{t} \ddot{u} \sin \left(\omega_{n}(t-T)\right) \mathrm{d} T, \\
\ddot{u} & =\left[\begin{array}{llll}
C_{1} & C_{2} & C_{3} & C_{4}
\end{array}\right]^{T},
\end{aligned}
$$

where $C_{i}$ is the constant step input. Substituting the piecewise function $\ddot{u}$ in equation (4b) gives

$$
\theta(t)=\frac{1}{\omega_{n} l} \sum_{i=1}^{4} \int_{(i-1) \Delta t}^{i \Delta t} C_{i} \sin \left(\omega_{n}(t-T)\right) \mathrm{d} T .
$$

Performing the integral reduces equation (5) to

$$
\theta(t)=\frac{1}{\omega_{n}^{2} l} \sum_{i=1}^{4} C_{i}\left(\cos \left(\omega_{n}(t-i \Delta t)\right)-\cos \left(\omega_{n}(t-(i-1) \Delta t)\right)\right) .
$$




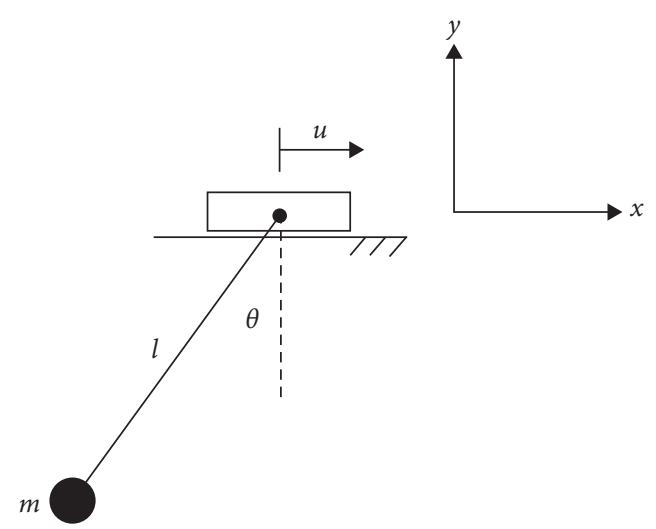

FIgURE 1: Overhead crane model with a massless slider.

Differentiating equation (6a) gives the angular velocity as

$$
\dot{\theta}(t)=\frac{1}{\omega_{n} l} \sum_{i=1}^{4} C_{i}\left(-\sin \left(\omega_{n}(t-i \Delta t)\right)+\sin \left(\omega_{n}(t-(i-1) \Delta t)\right)\right) .
$$

Substituting the accelerating-phase final-time $(\tau=4 \Delta t)$ into equations (6a) and (6b) gives

$$
\begin{gathered}
\theta(\tau)=\frac{1}{g} \sum_{i=1}^{4} C_{i}\left(\left(\cos \left((4-i) \omega_{n} \Delta t\right)-\cos \left((5-i) \omega_{n} \Delta t\right)\right)\right), \\
\dot{\theta}(\tau)=\frac{1}{\omega_{n} l} \sum_{i=1}^{4} C_{i}\left(\left(-\sin \left((4-i) \omega_{n} \Delta t\right)+\sin \left((5-i) \omega_{n} \Delta t\right)\right)\right) .
\end{gathered}
$$

Equations (7a) and (7b) represent the final angular position and final angular velocity of the mass $m$. The system final conditions and constraints are given by

$$
\begin{gathered}
\theta(\tau)=0, \\
\dot{\theta}(\tau)=0 . \\
\dot{u}(\tau)=v_{f},
\end{gathered}
$$

where $v_{f}$ is the desired speed of the jib at the end of the accelerating phase at $t=\tau$. Equations (8a) and (8b) impose two final conditions and one constraint to the system, which implies that three inputs are required to satisfy these requirements. However, an extra input is introduced here as the fourth constant to optimize the system performance according to a tailored objective function. This additional independent input provides the required adaptation to the system requirements according to the optimization criterion. By applying equations (8a) and (8b) to equations (7a) and $(7 b)$, the matrix representation of the system's conditions and constraint is found as

$$
\begin{aligned}
M_{1} \ddot{u} & =M_{2}, \\
M_{1} & =\left[\begin{array}{llll}
\alpha_{1} & \alpha_{2} & \alpha_{3} & \alpha_{4} \\
\beta_{1} & \beta_{2} & \beta_{3} & \beta_{4} \\
1 & 1 & 1 & 1
\end{array}\right], \\
M_{2} & =\left[\begin{array}{c}
0 \\
0 \\
v_{f} \\
\Delta t
\end{array}\right], \\
\alpha_{i} & =\left(\cos \left((4-i) \omega_{n} \Delta t\right)-\cos \left((5-i) \omega_{n} \Delta t\right)\right), \\
\beta_{i} & =\left(-\sin \left((4-i) \omega_{n} \Delta t\right)+\sin \left((5-i) \omega_{n} \Delta t\right)\right), \\
i & =1,2,3,4 .
\end{aligned}
$$

Rearranging equation (9) gives

$$
\begin{aligned}
{\left[\begin{array}{l}
C_{1} \\
C_{2} \\
C_{3}
\end{array}\right] } & =M_{3}^{-1}\left(M_{2}-M_{4} C_{4}\right), \\
M_{3} & =\left[\begin{array}{ccc}
\alpha_{1} & \alpha_{2} & \alpha_{3} \\
\beta_{1} & \beta_{2} & \beta_{3} \\
1 & 1 & 1
\end{array}\right], \\
M_{4} & =\left[\begin{array}{c}
\alpha_{4} \\
\beta_{4} \\
1
\end{array}\right] .
\end{aligned}
$$

Equation (10) represents the first three dependent inputs, which are the first three piecewise acceleration steps of the jib. These steps satisfy the system requirements given by using equations (8a) and (8b) 


\section{Results and Discussion}

3.1. Sensitivity Analysis. The sensitivity index (SI) is defined as follows to quantify the sensitivity of the vibration amplitude of the system to the cable length:

$$
\mathrm{SI}=\frac{1}{N} \sum_{i=1}^{N} \mathrm{Amp}_{i}
$$

where $\mathrm{Amp}_{i}$ is the amplitude of vibration at the end of the maneuver case $i$. A range of $80 \%$ to $120 \%$ of the cable length is used to calculate the sensitivity index at each point.

Figure 2 shows the overhead crane payload angle for a specific cable length. The system requirements are met by applying the step input generated by the proposed input shaper. The designated maneuver ends with zero payload angle and zero angular velocity, suggesting that the generated input steps eliminate the residual vibrations while the jib reaches the required final velocity.

Figure 3 shows how the sensitivity index changes by changing the fourth input constant $\left(C_{4}\right)$ for different cable lengths calculated at the standard maneuver time, which is 1 second. The results indicate that the effect of the fourth input constant on the sensitivity index increases as the cable length decreases. For a given range of cable lengths, there are optimum $C_{4}$ values for which the sensitivity index is minimized and the system is least sensitive to unexpected cable length variations.

Figure 4 shows the values of all input constants measured at the optimal $C_{4}$ and how they change as cable length changes. As the cable length increases, higher input constants are expected due to higher kinetic and potential energies of the system as the pendulum oscillates. It is also apparent that the input constants change in pairs: $C_{1}$ with $C_{4}$ and $C_{2}$ with $C_{3}$. At $L \approx 0.25 \mathrm{~m}$, all input constants are equal, suggesting that the system reaches the zero-vibration final state with a constant input throughout the maneuver. For a given cable length value, selecting the associated optimized input constants (plotted in Figure 4) guarantees that the system remains least sensitive to any unexpected cable length change within a variation range of $80 \%$ to $120 \%$.

Figure 5 shows how the sensitivity index changes for different cable lengths and a range of maneuver times. For a specific cable length, increasing the maneuver time decreases the system sensitivity to unexpected cable length variations. This observation is valid for all longer cable length values. Even for short cables $(L \leq 0.2 \mathrm{~m})$, in which SI decreases at the beginning of the maneuver due to a reduction in the natural frequency of the system, SI increases as the maneuver continues.

A new metric is introduced to normalize the impact of the maneuver time on the sensitivity index. The normalized sensitivity index $(\psi)$ is defined as the ratio of the sensitivity index for a given cable length and maneuver time to the sensitivity index with the standard maneuver time but similar cable length. Figure 6 shows how the normalized sensitivity index changes with the change of maneuver time for different cable lengths. Values less than one indicate that the system is less sensitive compared to the system's

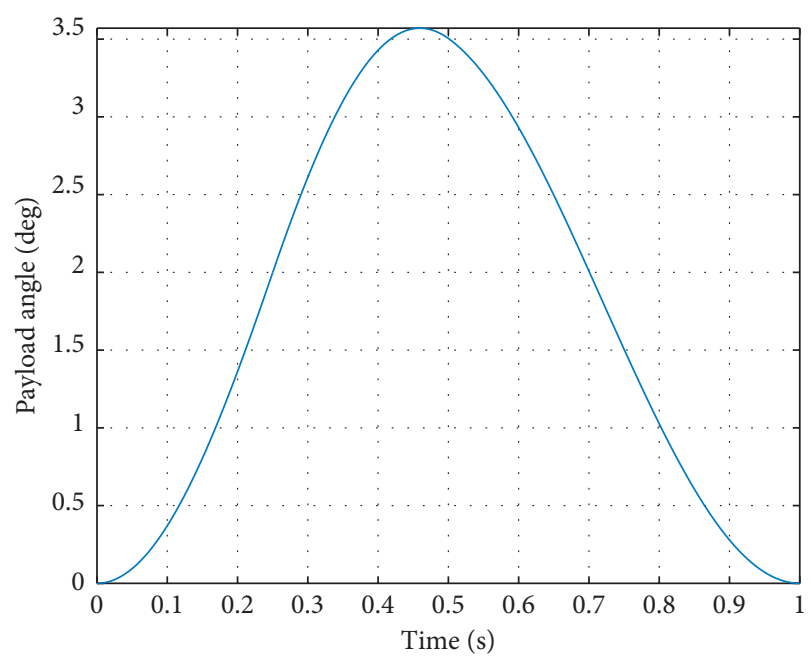

FIgURE 2: Payload angle versus maneuver time for a specific cable length.



FIgURE 3: Sensitivity index over different cable lengths and input values $\left(C_{4}\right)$ with the standard maneuver time.

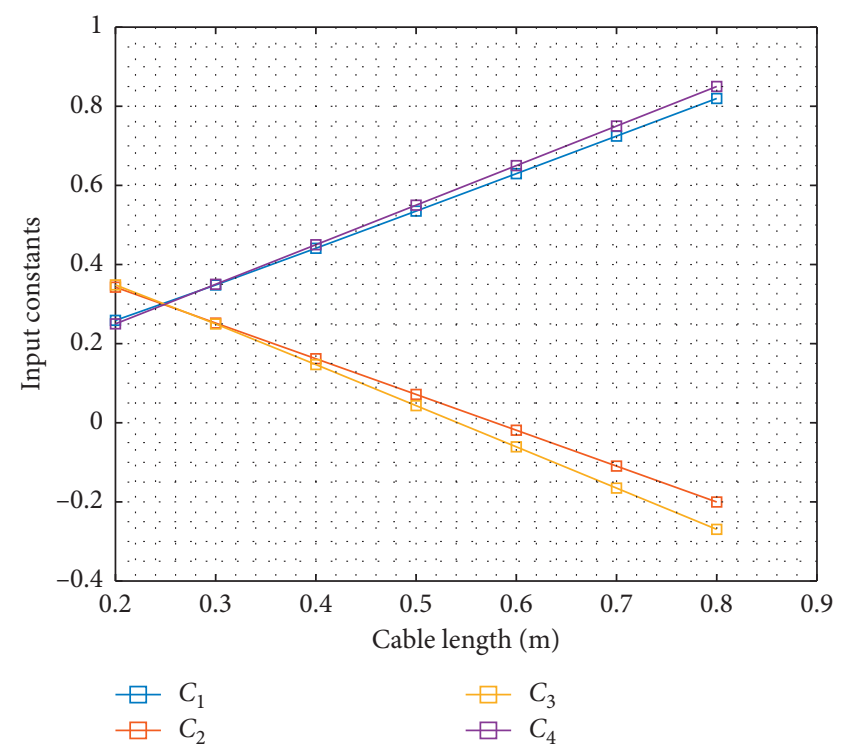

FIgURE 4: Input constant values versus cable lengths for the standard maneuver time. 


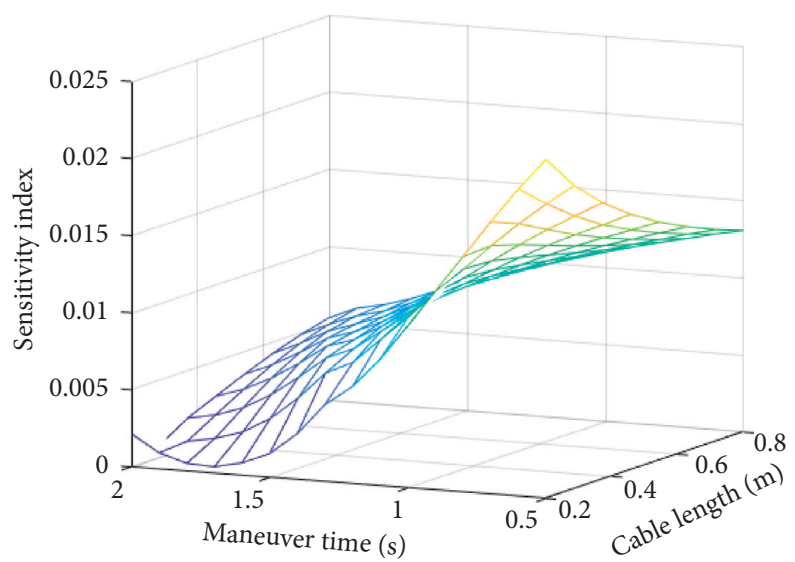

Figure 5: Sensitivity index over different cable lengths and a range of maneuver times.



Figure 6: Normalized sensitivity index $(\psi)$ over different cable lengths and a range of maneuver times.

sensitivity at the standard maneuver time for the same cable length. As shown in Figure 6, there are instances where the system is less sensitive and the maneuver time is shorter, suggesting that the proposed approach can reduce the maneuver time for a given cable length at the same time that it reduces the sensitivity.

3.2. Jerk Analysis. Significant differences between input values may be unfavorable due to the existence of a jerk. Therefore, jerk index (JI) is introduced to measure these differences as follows:

$$
\begin{aligned}
\mathrm{JI} & =\sqrt{\sum_{i=1}^{5}\left(C_{i}-C_{i-1}\right)^{2}}, \\
C_{0} & =C_{5}=0 .
\end{aligned}
$$

Figure 7 shows the relationship between the jerk index of the system and the fourth input constant $\left(C_{4}\right)$ for different cable length settings. For a given cable length, there is an optimum value of $C_{4}$, for which the jerk index of the system

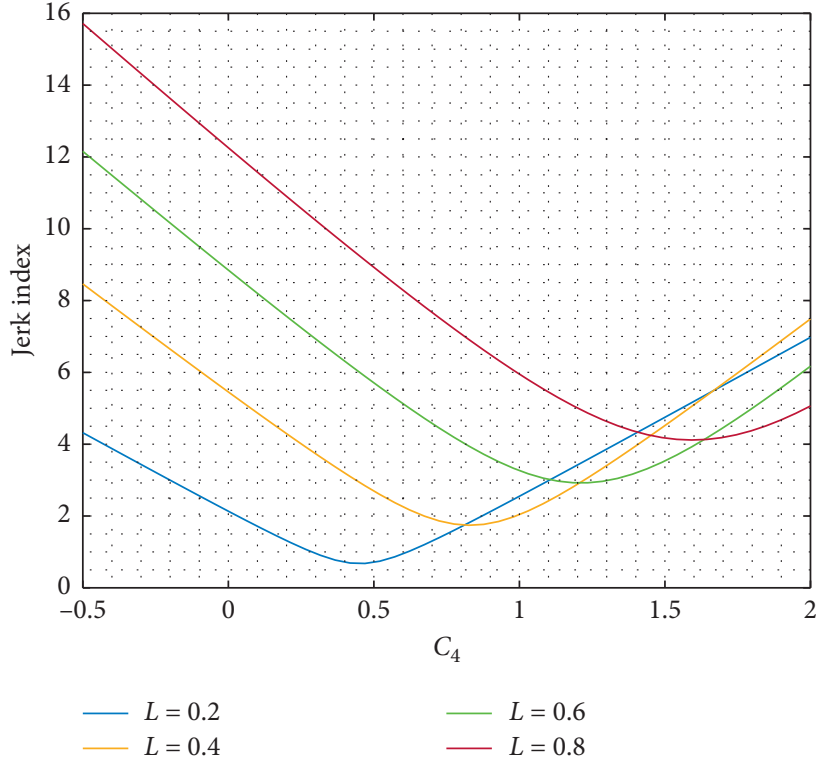

Figure 7: Jerk index versus the fourth input constant $\left(C_{4}\right)$ for different cable lengths with the standard maneuver time.

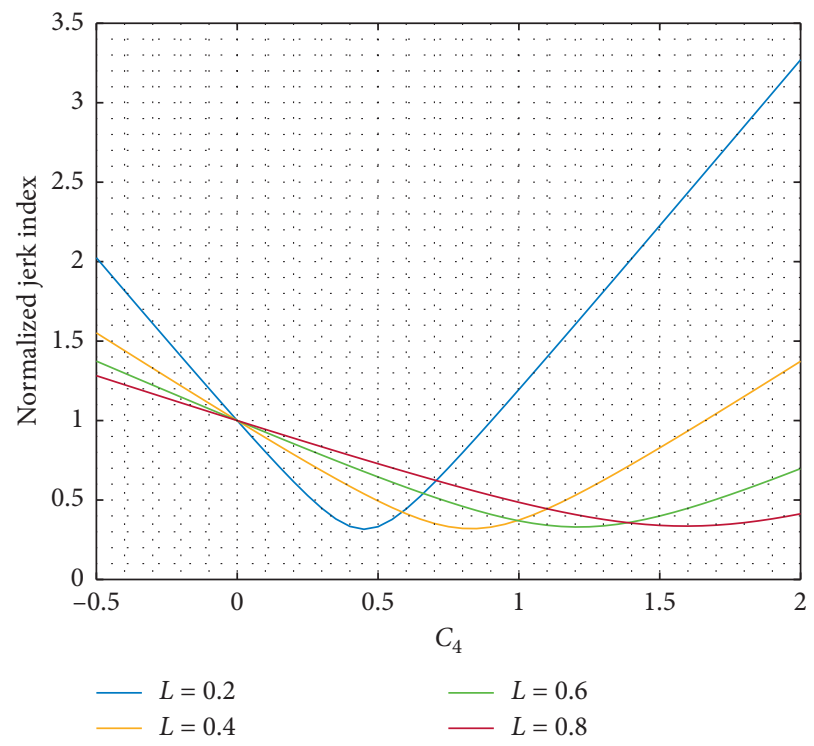

FIGURE 8: Normalized jerk index versus the fourth input constant $\left(C_{4}\right)$ for different cable lengths with the standard maneuver time.

is minimized. Additionally, as expected, increasing the cable length increases the total energy of the system which results in a higher jerk index and optimal $C_{4}$ value.

Normalized jerk index (NJI) is defined as the ratio of the jerk index to the jerk index with zero $C_{4}$ for the same cable length as follows:

$$
\mathrm{NJI}=\frac{\mathrm{JI}}{\left.\mathrm{JI}\right|_{C_{4}=0}} .
$$

NJI indicates how the jerk index is affected by adding $C_{4}$ to the formulation. Figure 8 shows the normalized jerk index and its relationship with $C_{4}$ for different cable lengths. 


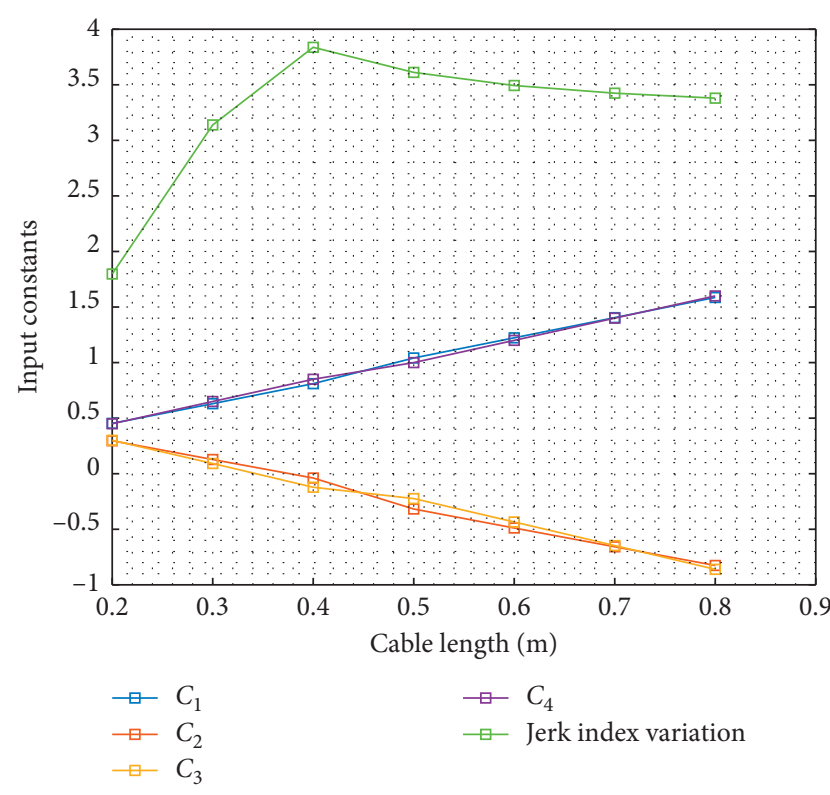

FIGURE 9: Input constants and jerk index variation versus cable length for the standard maneuver time.

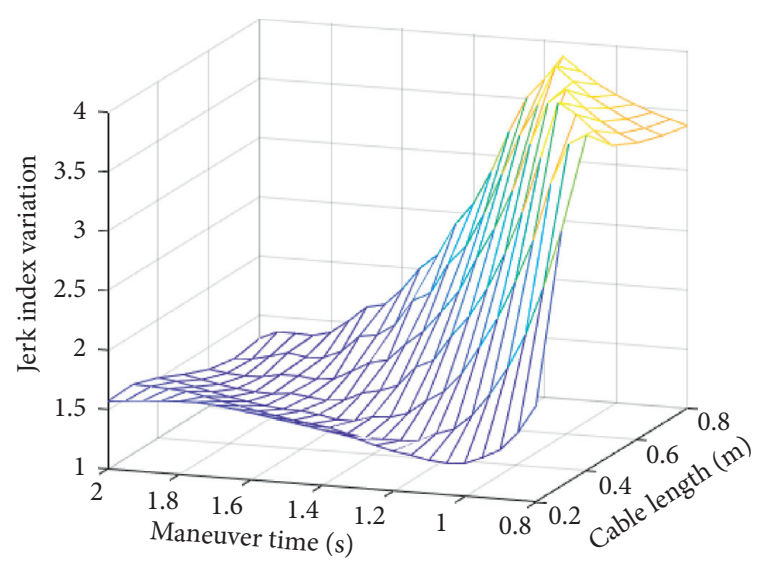

FIGURE 10: Jerk index variation over different maneuver times and cable lengths.

Values less than one indicate that the system is improved by adding $C_{4}$ compared to the system with three input constants (i.e., $C_{4}=0$ ). Increasing the cable length extends the range at which the normalized jerk index is less than one.

Jerk index variation (JIV) is introduced as the ratio of the jerk index to the mean of absolute values of the input constant:

$$
\mathrm{JIV}=\frac{\mathrm{JI}}{(1 / 4) \sum_{i=1}^{4} C_{i}} .
$$

Figure 9 shows the input constants calculated at the optimum $\mathrm{C}_{4}$ to minimize the jerk index for different cable lengths. Higher values of jerk index variation indicate that the jerks are unfavorably high relative to the input constants.

Figure 10 shows how the jerk index variation changes by changing the cable length over a range of maneuver times. In most cases, increasing the maneuver time results in the reduction of the jerk index while it also reduces the deviation between the input constants. The jerk index has the highest values for long cables with short maneuver times. A system with this configuration is challenging to control as it requires large variations among the inputs.

\section{Conclusions}

This paper presented a novel input shaping approach to address the limitations of conventional $\mathrm{ZV}$ shaping techniques in controlling overhead cranes with demanding design and operational requirements. The presented method results in optimal maneuver time with a minimal jerk and parameter insensitivity. An extensive sensitivity analysis is conducted to quantify the performance of the controller under different feasible operating conditions. The jerk analysis indicates that the optimization process by using the shaper enables it to reduce the input jerk while minimizing maneuver time. The results confirm that the modified ZV shaper provides a powerful yet effective and computationally efficient control solution for overhead cranes with demanding point-to-point maneuver requirements.

\section{Data Availability}

No data were used to support this study.

\section{Conflicts of Interest}

The authors declare that they have no conflicts of interest.

\section{References}

[1] H. Butler, G. Honderd, and J. Van Amerongen, "Model reference adaptive control of a gantry crane scale model," IEEE Control Systems, vol. 11, no. 1, pp. 57-62, 1991.

[2] R. Manning, J. Clement, D. Kim, and W. Singhose, "Dynamics and control of bridge cranes transporting distributed-mass payloads," Journal of Dynamic Systems Measurement and Control, vol. 132, no. 1, p. 14505, 2010.

[3] E. M. Abdel-Rahman, A. H. Nayfeh, and Z. N. Masoud, "Dynamics and control of cranes: a review," Journal of $\mathrm{Vi}$ bration and Control, vol. 9, no. 7, pp. 863-908, 2003.

[4] K. C. C. Peng, W. Singhose, and D. H. Frakes, "Hand-motion crane control using radio-frequency real-time location systems," IEEE/ASME Transactions Mechatronics, vol. 17, no. 3, pp. 464-471, 2012.

[5] J. Yu, F. L. Lewis, and T. Huang, "Nonlinear feedback control of a gantry crane," in Proceedings of the 1995 American Control Conference, pp. 4310-4315, IEEE, Seattle, WA, USA, June 1995.

[6] K. A. F. Moustafa, "Feedback control of overhead cranes swing with variable rope length," in Proceedings of the 1994 American Control Conference, pp. 691-695, IEEE, Baltimore, MD, USA, July 1994.

[7] H. Chen and N. Sun, "Nonlinear control of underactuated systems subject to both actuated and unactuated state constraints with experimental verification," IEEE Transactions on Industrial Electronics, vol. 67, no. 9, pp. 7702-7714, 2019.

[8] A. Gholabi, M. Ebrahimi, G. R. Yousefi, M. Ghayour, A. Ebrahimi, and H. Jali, "Sensorless anti-swing control for 
overhead crane using voltage and current measurements," Journal of Vibration and Control, vol. 21, no. 9, pp. 1745-1756, 2015.

[9] J. Smoczek and J. Szpytko, "Evolutionary algorithm-based design of a fuzzy TBF predictive model and TSK fuzzy antisway crane control system," Engineering Applications of Artificial Intelligence, vol. 28, pp. 190-200, 2014.

[10] H. Chen, Y. Fang, and N. Sun, "An adaptive tracking control method with swing suppression for 4-DOF tower crane systems," Mechanical Systems and Signal Processing, vol. 123, pp. 426-442, 2019.

[11] M. Zhang, X. Ma, X. Rong, X. Tian, and Y. Li, "Error tracking control for underactuated overhead cranes against arbitrary initial payload swing angles," Mechanical Systems and Signal Processing, vol. 84, pp. 268-285, 2017.

[12] T. Yang, N. Sun, H. Chen, and Y. Fang, "Neural networkbased adaptive antiswing control of an underactuated shipmounted crane with roll motions and input dead zones," IEEE Transactions on Neural Networks and Learning Systems, vol. 31, no. 3, pp. 901-914, 2019.

[13] R. Tang and J. Huang, "Control of bridge cranes with distributed-mass payloads under windy conditions," Mechanical Systems and Signal Processing, vol. 72-73, pp. 409-419, 2016.

[14] H. Chen, B. Xuan, P. Yang, and H. Chen, "A new overhead crane emergency braking method with theoretical analysis and experimental verification," Nonlinear Dynamics, vol. 98, no. 3, pp. 2211-2225, 2019.

[15] D. Schindele and H. Aschemann, "Fast nonlinear MPC for an overhead travelling crane," IFAC Proceedings Volumes, vol. 44, no. 1, pp. 7963-7968, 2011.

[16] J. Smoczek and J. Szpytko, "Soft-constrained predictive control for an overhead crane," Journal of KONES, vol. 24, 2017.

[17] M. Giacomelli, M. Faroni, D. Gorni, A. Marini, L. Simoni, and A. Visioli, "MPC-PID control of operator-in-the-loop overhead cranes: a practical approach," in Proceedings of the 7th International Conference on Systems and Control, pp. 321326, IEEE, Valencia, Spain, October 2018.

[18] N. C. Singer, W. P. Seering, and K. A. Pasch, "Shaping command inputs to minimize unwanted dynamics," U. S. Patent 4916635A, MIT Press, Cambridge, MA, USA, 1990.

[19] N. C. Singer and W. P. Seering, "Preshaping command inputs to reduce system vibration," Journal of Dynamic Systems, Measurement and Control, vol. 112, no. 1, pp. 76-82, 1990.

[20] D. Kim and W. Singhose, "Performance studies of human operators driving double-pendulum bridge cranes," Control Engineering Practice, vol. 18, no. 6, pp. 567-576, 2010.

[21] M. A. Ahmad, R. M. T. R. Ismail, M. S. Ramli, N. M. A. Ghani, and N. Hambali, "Investigations of feed-forward techniques for anti-sway control of 3-D gantry crane system," in Proceedings of the 2009 IEEE Symposium on Industrial Electronics Applications, pp. 265-270, IEEE, Kuala Lumpur, Malaysia, October 2009.

[22] S. Garrido, M. Abderrahim, A. Giménez, R. Diez, and C. Balaguer, "Anti-swinging input shaping control of an automatic construction crane," IEEE Transactions on Automation Science Engineering, vol. 5, no. 3, pp. 549-557, 2008.

[23] M. Gniadek, "Usage of input shaping for crane load oscillation reduction," in Proceedings of the 20th International Conference on Methods Model Automations Robotics, pp. 278-282, IEEE, Miedzyzdroje, Poland, August 2015.

[24] T. Singh, "Jerk limited input shapers," in Proceedings of the American Control Conference, pp. 4825-4830, IEEE, Boston, MA, USA, 2004.
[25] J. Vaughan, A. Yano, and W. Singhose, "Comparison of robust input shapers," Journal of Sound and Vibration, vol. 315, no. 4, pp. 797-815, 2008.

[26] K. Alghanim, A. Mohammed, and M. T. Andani, "An input shaping control scheme with application on overhead cranes," International Journal of Nonlinear Science and Numerical Simulation, vol. 20, no. 5, pp. 561-573, 2019.

[27] M. J. Maghsoudi, Z. Mohamed, A. R. Husain, and M. O. Tokhi, "An optimal performance control scheme for a 3D crane," Mechanical Systems and Signal Processing, vol. 6667, pp. 756-768, 2016.

[28] A. Mohammed, K. Alghanim, and M. T. Andani, "An optimized non-linear input shaper for payload oscillation suppression of crane point-to-point maneuvers," International Journal Dynamics and Control, vol. 7, no. 2, p. 567, 2019.

[29] L. Ramli, Z. Mohamed, and H. I. Jaafar, "A neural networkbased input shaping for swing suppression of an overhead crane under payload hoisting and mass variations," $\mathrm{Me}$ chanical Systems and Signal Processing.vol. 107, pp. 484-501, 2018.

[30] A. M. Abdullahi, Z. Mohamed, H. Selamat et al., "Adaptive output-based command shaping for sway control of a 3D overhead crane with payload hoisting and wind disturbance," Mechanical Systems Signal Processing, vol. 98, pp. 157-172, 2018. 